\title{
Analysis of Mechanical Fixation Made of Aluminum Alloy in an Axial Flux Permanent Magnet Machine
}

\author{
Jiyoung Lee*, Byounggung Park, and Daehyun Koo \\ Electric Motor Research Center, Korea Electrotechnology Research Institute, 12, Bulmosan-ro 10 beon-gil, \\ Seongsan-go, Changwon 642-120, Korea
}

(Received 31 July 2014, Received in final form 18 August 2014, Accepted 19 August 2014)

\begin{abstract}
This paper presents an eddy current loss analysis of a Mechanical Fixation (MF) made of 6061 aluminum alloy, which is used for an NS type double-rotor single-stator axial flux permanent magnet machine. The prototype MF made of aluminum alloy shows good mechanical performance, but poor electro-magnetic performance, since the machine's efficiency can decrease because of eddy current loss in the MF. In order to prevent efficiency decrease, a modification of the MF structure is also introduced. Three-dimensional finite element analysis (FEA) is used for magnetic field analysis, and eddy current losses are computed. The analysis results are compared to, and verified by the test results.
\end{abstract}

Keywords : axial flux machine, double-rotor, eddy current loss, mechanical fixation

\section{Introduction}

The axial flux permanent magnet (AFPM) machine demonstrates distinct advantages of large power density and compact construction over the traditional radial-flux permanent magnet (RFPM) machine, due to better flux utilization, and improved cooling $[1,2]$. Therefore in this research, the AFPM machine was selected for the generator of a portable generating system to charge a 100 Wh lithium-polymer battery, due to its advantage of high power density. The portable generating system is for battery charging application in battle-field situations, or backcountry. A gear system and an AFPM generator are incorporated to further minimize the total mass and space for the portable generating system, and soft magnetic composite (SMC) cores are used in the AFPM generator, instead of silicon steel cores. The basic composition of the generating system is the same as the generating system in [3].

Among several multi-stage topologies [4-6], NS type double-rotor single-stator (DR-SS) topology is used in the AFPM generator of this research. NS type refers to northpole and south-pole magnets facing each other at either

CThe Korean Magnetics Society. All rights reserved.

*Corresponding author: Tel: +82-55-280-1416

Fax: +82-55-280-1490, e-mail: jylee@keri.re.kr side of the stator; thus the flux can travel straight through the stator, without any circumferential flow. This NS type DR-SS topology can have a higher power density than other multi-stage topologies, because the stator yoke is dispensable [6]. Correctly speaking, the stator yoke is not needed as a magnetic flux path, but a similar structure to the stator yoke is required for mechanical fixing. Therefore the design of the mechanical fixation (MF) of the stator in NS type DR-SS topology is a very important part in the overall motor design process.

The volume of the MF in NS type DR-SS topology must be smaller than the stator volume of other multistage topologies with stator yoke, to retain the advantage of no stator yoke. Furthermore, the MF should be made of non-electric and non-magnetic conducting material of high strength, in order to not reduce the efficiency. However, when considering the fabrication cost, it is difficult to find material that satisfies both the electro-magnetic characteristics of non-electric and non-magnetic conduction, and the mechanical characteristic of high strength.

This paper presents an eddy current loss analysis for an MF made of 6061 aluminum alloy in an NS type DR-SS AFPM generator. The MF is in the shape of a pair of wheels with many spokes, in order to fix tooth-tips, not the middle of each tooth, as shown in Figs. 1, 2, and 4. The axial length and overall volume can be reduced because of this configuration. 


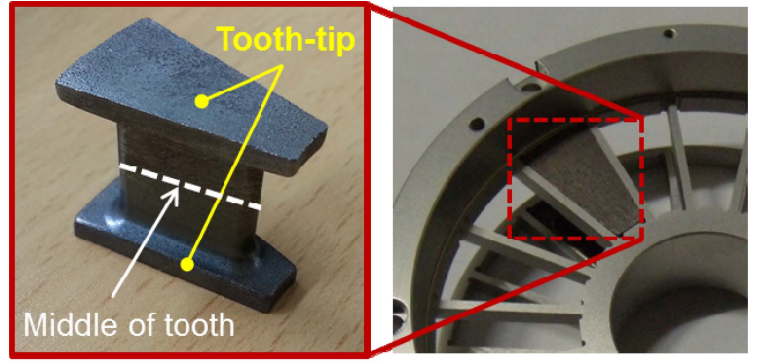

(a) stator tooth

(b) MF inserted one stator tooth

Fig. 1. (Color online) The configurations of a stator tooth and an MF for the NS type DR-SS AFPM generator.
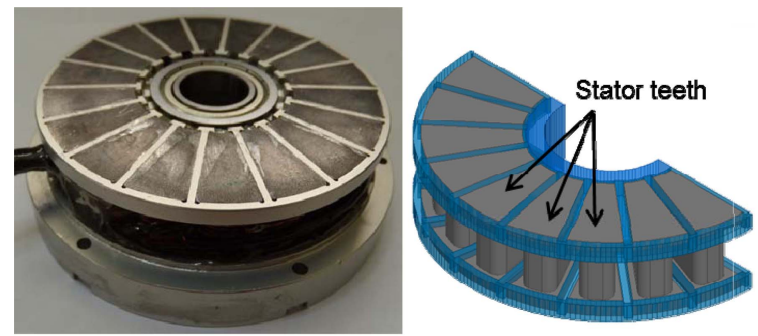

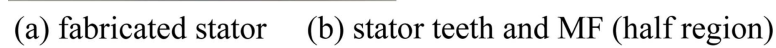

Fig. 2. (Color online) The fabricated and analysis models for stator teeth and the MF.

6061 aluminum alloy is selected, because the mechanical performances per price are satisfactory, in terms of strength and weight. However, the eddy currents in the MF made of aluminum alloy can reduce the magnetic performance of the machine. In order to prevent efficiency decrease, a modification of the MF structure is introduced, and its eddy current loss is compared with the loss of the prototype MF that is designed by considering just mechanical performances. Three-dimensional (3-D) finite element analysis (FEA) is used for magnetic field analysis [7], and eddy current losses are computed. The analysis results are compared to, and verified by the test results.

\section{Analysis models}

\subsection{Specifications and two analysis models}

For the NS type DR-SS AFPM generator, Table 1 lists the main analysis specifications, and Figs. 2 and 3 show the analysis models. In order to present the detailed configurations of stator teeth and the MF, Fig. 2(b) shows a partial analysis model. Fig. 3 shows the overall analysis models.

It takes much time to get steady state results for loss calculation from the 3-D FEA in the time domain. In order to save computation time for the many analyses of the variations of MF configuration, 3-D FEA in the fre-

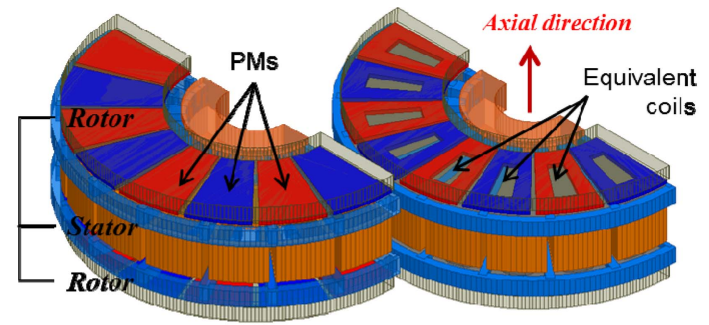

(a) the time domain model (b) the frequency domain model

Fig. 3. (Color online) Analysis models of the NS type DR-SS AFPM generator (half region, with periodic boundary conditions).

quency domain was used with several assumptions, and the analysis results obtained through a single 3-D FEA in the time domain. Therefore, Fig. 3 presents the two analysis models. Fig. 3(a) is the time domain model, and practical materials and shapes are used and depicted for cores, PMs, coils, and the MF. Fig. 3(b) is the frequency domain model, and the PM is converted into the equivalent coils. The detailed differences of the two analysis models will be explained in the next section about the analysis method.

\subsection{Mechanical fixation}

As the MF is a part of the stator, the same material as listed in Table 1 is used in both the time and frequency domain analysis models. However, variable configurations are examined, to find the proper shape that minimizes the eddy current loss.

Figs. 4 and 5 show the different configurations of the MFs, depending on the presence of slits. The MF in Fig. 4 is termed the 'prototype MF' in this paper. The MF consists of inner-ring, spokes, and outer ring. The innerring is linked to the axis by bearings, and the outer-ring is fixed to a housing. The spokes keep the intervals of the stator tooth-tips. To reduce eddy current loss, slits are introduced in both the upper- and undersides of the innerring, as shown in Fig. 5.

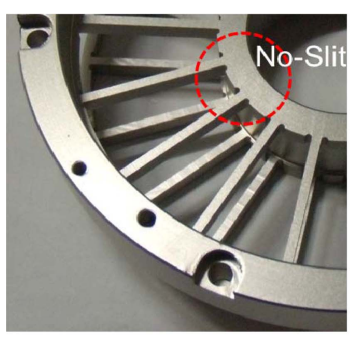

(a) fabricated model

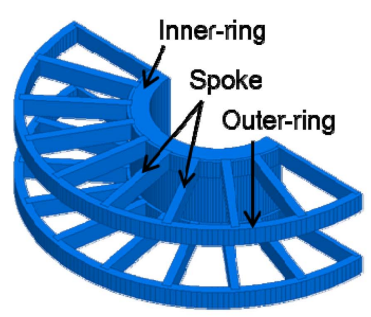

(b) analysis model
Fig. 4. (Color online) The fabricated and analysis models for the prototype MF without slit. 


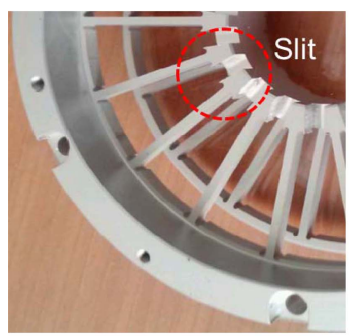

(a) fabricated model

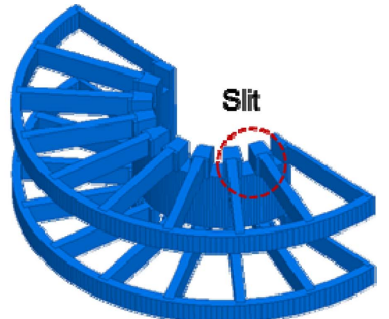

(b) analysis model
Fig. 5. (Color online) The fabricated and analysis models for the MF with slits (one side slit depth=4 mm).

Table 1. The common analysis specifications of the NS type DR-SS AFPM generator.

\begin{tabular}{lc}
\hline \multicolumn{1}{c}{ Contents } & Values \\
\hline $\begin{array}{l}\text { Rated speed/Rated frequency of induced } \\
\text { voltage }\end{array}$ & $1800 \mathrm{rpm} / 240 \mathrm{~Hz}$ \\
Rated induced phase voltage & $17 \mathrm{Vrms}$ \\
No. of pole/slot & $16 / 18$ \\
Magnetic core material & Somaloy700-1P \\
PM material & $\mathrm{N} 38 \mathrm{UH}(\mathrm{Br}=1.23 \mathrm{~T})$ \\
Mechanical fixation material, and its & $\mathrm{Al} 6061,2.32 \times 10^{7} \mathrm{~S} / \mathrm{m}$ \\
conductivity & \\
Stator axial length & $20 \mathrm{~mm}$ \\
PM thickness & $1 \mathrm{~mm}$ \\
Overall outer diameter & $100 \mathrm{~mm}$ \\
\hline
\end{tabular}

\section{Analysis Method and the Results}

\subsection{Analysis method}

Time domain and frequency domain FEAs of the 3D electromagnetic field in each analysis model in Fig. 3 were performed, which were based on the $\mathrm{T}-\Omega$ formulation. The related governing equations are introduced in $[8,9]$. For the two FEA solvers, Table 2 compares the general pre-process conditions and solving-times of each model shown in Fig. 3.

As mentioned before, multiple time frequency domain analyses were performed after one time domain analysis. The main four assumptions for the frequency domain are as follows:

1) Motion: instead of motional speed, the electromagnetic fields of PMs pulsating with specific frequency are considered.

2) Excitations: the magneto-motive force ( $\mathrm{mmf}$ ) of the PM is calculated as in (1), and used as the external current source of the model, as shown in Fig. 3(b). In the no-load condition, the mmf of the PM is the only source for eddy current loss computation.

$$
\Gamma=l_{m} \times B_{r} / \mu_{0} \mu_{r}
$$

where, $\Gamma$ is the mmf of the PM, $l_{m}$ is the magnetization direction length of the PM, $B_{r}$ is the residual flux density, $\mu_{0}$ is the space permeability, and $\mu_{r}$ is the relative permeability of PM.

3) Source shape: the shape of the exciting current source is a sine wave alternating current. The magnitude is calculated as in (1). The source frequency is decided as the interested speed, considering the rated speed of the machine.

4) Material: instead of one non-linear magnetic material for cores, several linear materials are used. After time domain analysis, average flux densities are calculated for the main three core parts of rotor yoke, stator tooth tip, and stator tooth. From the average flux densities of the three parts, the relative permeability of Somaloy $700-1 \mathrm{P}$ is calculated, and used for the linear materials.

\subsection{Analysis results}

The eddy current analysis was performed for the noload generating condition, when the frequency of induced voltage, which is the source frequency, was from $40 \mathrm{~Hz}$ to $240 \mathrm{~Hz}$ with $40 \mathrm{~Hz}$ interval. The slit depth of MF was changed from $0 \mathrm{~mm}$ to $10 \mathrm{~mm}$, with 4 points - $0,4,8,10$ $(\mathrm{mm})$. A totally of 24 frequency domain analyses were made. Table 3 lists the analysis specifications for source and material conditions.

Table 2. A comparison of the time domain and frequency domain FEAs.

\begin{tabular}{ccc}
\hline \hline Contents & Time domain (transient solver) & Frequency domain (eddy current solver) \\
\hline Motion & Allowed & Not allowed \\
Excitations & Current, Voltage, PM & Current only \\
Source shape & Functions of time & Functions of Frequency \\
Nonlinear Material & Allowed & Not allowed \\
Boundary Conditions for this research & Neumann, Matching (Master-slave) & Neumann, Matching (Master-slave), \\
Calculated results for this research & Induced voltage Flux density Core loss & Zero tangential H field \\
Calculation time for one speed (or frequency) & About 24 hours & Eddy current and its ohmic loss \\
\hline
\end{tabular}


Table 3. The analysis specifications for eddy current analysis (frequency domain solver).

\begin{tabular}{ll}
\hline \hline Contents & Values \\
\hline mmf of the PM & 932.2 AT \\
Relative permeability of the & 511.8 for rotor yoke, 533 for stator \\
cores & tooth and tooth-tip \\
Source frequency & $40 \mathrm{~Hz}$ interval, until $240 \mathrm{~Hz}$
\end{tabular}

Table 4. Eddy current losses according to slit depth and frequency.

\begin{tabular}{|c|c|c|c|c|}
\hline \multirow{2}{*}{$\begin{array}{l}\text { Frequency } \\
\text { (Generating speed) }\end{array}$} & \multicolumn{4}{|c|}{ Eddy current loss (W) } \\
\hline & $0 \mathrm{~mm}$ & $4 \mathrm{~mm}$ & $8 \mathrm{~mm}$ & $10 \mathrm{~mm}$ \\
\hline $40 \mathrm{~Hz}(300 \mathrm{rpm})$ & 9.95 & 9.04 & 8.36 & 0.01 \\
\hline $80 \mathrm{~Hz}(600 \mathrm{rpm})$ & 38.12 & 33.62 & 31.26 & 0.03 \\
\hline $120 \mathrm{~Hz}(900 \mathrm{rpm})$ & 80.15 & 69.40 & 65.08 & 0.08 \\
\hline $160 \mathrm{~Hz}(1200 \mathrm{rpm})$ & 130.58 & 111.59 & 105.76 & 0.13 \\
\hline $200 \mathrm{~Hz}(1500 \mathrm{rpm})$ & 184.31 & 155.96 & 149.50 & 0.21 \\
\hline $240 \mathrm{~Hz}(1800 \mathrm{rpm})$ & 237.50 & 199.46 & 193.35 & 0.30 \\
\hline
\end{tabular}

Fig. 6 shows, and Table 4 lists the analysis results, respectively. Fig. 6 compares the shapes of eddy current paths. The eddy current loop path changes, depending on the slit depth. When the slit depth is deeper, the length of eddy current path is longer, and the resistance is greater. If the inner-ring is cut because the slit depth is $10 \mathrm{~mm}$, as shown in Fig. 6(b), the eddy current path has the shortest length, but also has the narrowest width. This path has the greatest resistance.

Table 4 lists the ohmic losses of eddy current for the variations of slit depth and source frequency. The eddy current loss increases according to the source frequency, because the magnitude of the induced voltage in conductors increases. However, the eddy current loss decreases according to the slit depth, because the resistance increases, as explained in Fig. 6. When the slit depth is $10 \mathrm{~mm}$, the eddy current loss of the MF can be ignored. Therefore, the shape of the MF in Fig. 6(b) is highly recommended for the proper configuration of the MF made of aluminum
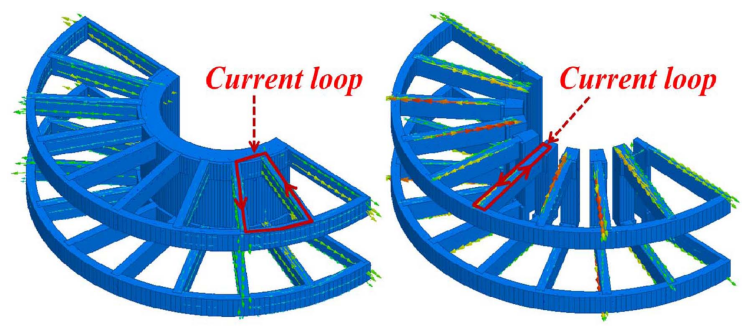

(a) the prototype MF

(b) the MF with $10 \mathrm{~mm}$ slit depth

Fig. 6. (Color online) The eddy current loop for each model.

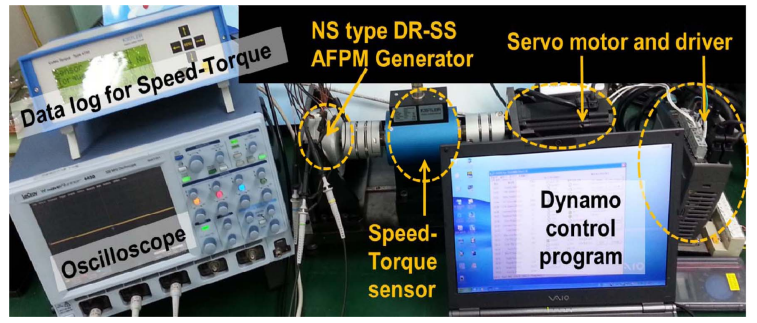

Fig. 7. (Color online) Test bed for power loss experiment.

alloy, in order to not reduce efficiency.

\section{Experiment Results}

In order to measure the losses, an NS type DR-SS AFPM generator is fabricated. The generator has an MF with $4 \mathrm{~mm}$ slit depth, as shown in Fig. 5. The test bed is set up as shown in Fig. 7. Because of the power limit in a dynamo servo-motor, the power losses were measured until $120 \mathrm{~Hz}$. At low speed, such as in this application, mechanical loss and eddy current loss on the PM can be ignored. Therefore, the measured no-load input power and the sum of the calculated losses in the MF and cores can be treated as the same value. The core losses, which are not dealt with in this paper, can be computed by using the method in [10]. The comparison of the calculated and measured losses of Fig. 8 shows that the computation results agree well with the measured ones, and the eddy current loss at the MF is dominant in the power losses.

\section{Conclusions}

Both time domain and frequency domain analyses were performed, in order to calculate the eddy current losses in the MF, considering time efficiency. The MF made of

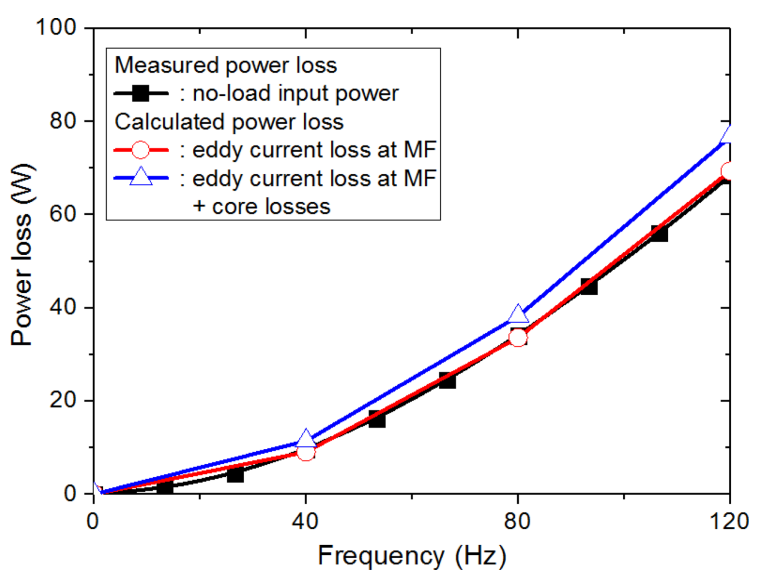

Fig. 8. (Color online) Comparison of power losses (slit depth $=4 \mathrm{~mm}$ ). 
aluminum alloy has enormous losses, as verified by analysis and the measured results, if there are no proper slits. When the slit depth is $10 \mathrm{~mm}$, the loss of the MF could be ignored. This research has shown an approach whereby eddy current losses can be rapidly and reliably evaluated, and also suggested a proper configuration of an MF made of aluminum alloy for an NS type DR-SS AFPM generator.

\section{Acknowledgement}

This work was supported by the Next Generation Military Battery Research Center program of the Defense Acquisition Program Administration and Agency for Defense Development.

\section{References}

[1] T. F. Chan, W. Wang, and L. L. Lai, IEEE Trans. Energy
Conversion 25, 669 (2010).

[2] F. Marignetti, V. D. Colli, and S. Carbone, IEEE Trans. Magn. 46, 598 (2010).

[3] J. Y. Lee, D. H. Koo, S. R. Moon, and C. K. Han, IEEE Trans. Magn. 48, 2977 (2012).

[4] M. Aydin, S. Huang, and T. A. Lipo, IEEE Trans. Ind. Electron. 53, 822 (2006).

[5] T. J. Woolmer and M. D. McCulloch, Electric Machine \& Drives Conference, IEMDC'07, (2007) pp. 704-708.

[6] T. J. Woolmer and M. D. McCulloch, Hybrid Vehicle Conference, IET, (2006) pp. 27-42.

[7] J. Y. Lee, D. H. Koo, D. H. Kang, and J. P. Hong, J. Magn. 17, 51 (2012).

[8] D. Lin, P. Zhou, and Q. M. Chen, IEEE Vehicle Power and Propulsion Conf. (VPPC), Harbin, China (2008).

[9] Maxwell Online Help, Ansoft Corporation, http:// www.ansoft.co.kr/.

[10] J. Y. Lee, S. R. Moon, D. H. Koo, D. H. Kang, G. H. Lee, and J. P. Hong, Journal of Electrical Engineering \& Technology 6, 350 (2011). 